\title{
DISPERSION RELATION AND BAND GAPS OF 3D PHOTONIC CRYSTALS MADE OF SPHERES
}

\author{
F. Güller ${ }^{1,2,{ }^{*}}$, M. E. Inchaussandague ${ }^{3}$, and R. A. Depine ${ }^{3}$ \\ ${ }^{1}$ Grupo de Materia Condensada, Centro Atómico Constituyentes, \\ GIyA, CNEA, Av. Gral. Paz 1499, San Martín, Buenos Aires, \\ Argentina \\ ${ }^{2}$ Consejo Nacional de Investigaciones Científicas y Técnicas, Av. Riva- \\ davia 1917, Buenos Aires, Argentina \\ ${ }^{3}$ Grupo de Electromagnetismo Aplicado, Departamento de Física, \\ FCEN, Universidad de Buenos Aires, Ciudad Universitaria, Pabellón I, \\ Buenos Aires, Argentina
}

\begin{abstract}
In this paper, we introduce a dispersion equation for 3D photonic crystals made of parallel layers of non-overlapping spheres, valid when both wavelength and separation between layers are much larger than the distance between neighbouring spheres. This equation is based on the Korringa-Kohn-Rostoker (KKR) wave calculation method developed by Stefanou et al. and can be used to predict the spectral positions of bandgaps in structures made of dispersive spheres. Perfect agreement between the spectral positions of bandgaps predicted with our simplified equation and those obtained with the numerical code MULTEM2 was observed. We find that this simplified relation allows us to identify two types of bandgaps: those related to the constitutive parameters of the spheres and those related to the three dimensional periodicity (distance between layers). Bandgaps of the first type are independent of the frequency and the distance between layers, while those of the second type depend only on these two quantities. We then analyze the influence of the constitutive parameters of the spheres on the spectral position of bandgaps for spheres immersed in dielectric or magnetic homogeneous media. The number and positions of the bandgaps are affected by the permitivity (permeability) of the host medium if the spheres have dispersive permitivity (permeability).
\end{abstract}

Received 14 May 2011, Accepted 17 June 2011, Scheduled 22 June 2011

* Corresponding author: Francisco Güller (guller@tandar.cnea.gov.ar). 


\section{INTRODUCTION}

The study of three-dimensional (3D) photonic crystals (PCs) has attracted a lot of attention in recent years and remains the focus of activity of many research groups. Photonic crystals exhibit potential applications in optical, infrared and microwave devices [1,2]. An interesting aspect of these materials is the existence of frequency regions, known as photonic bandgaps, over which propagation of light is forbidden $[3,4]$. This property has been proposed for several technological applications that are still under continuous research.

A typical example of 3D photonic crystal is a stack of layers formed by spherical particles arranged in a two dimensional periodic lattice. Among the methods suggested for the calculation of frequency bands in these kind of structures, the so-called on-shell methods appear to be numerically efficient and at the same time allow the calculation of the transmission/reflection coefficient. Electromagnetic interactions between the scatterers arranged in the periodic lattice are calculated by means of the layer-multiple-scattering method for spherical scatterers, usually known as the vector version of the Korringa-Kohn-Rostoker (KKR) method [5-7]. Recently, reflectance spectra simulated by adding extinction to the vector KKR method, have shown a clear correlation with the experimental results in the high energy range $[8,9]$.

To fully exploit the properties of 3D PCs made of spheres, we need to understand the origin of the photonic bandgap of the structure. Unfortunately, the dispersion relation for these systems is a non linear equation involving many variables and must be solved numerically. Therefore, it is a difficult task to investigate the influence of the relevant parameters of the structure (typical dimensions, permittivity and permeability of the media, etc.) on the spectral positions of the bandgaps. However, there are situations for which this equation can be simplified. Although even in these cases the dispersion equation must be solved numerically, its simpler form allows us to obtain additional information about the physical mechanisms that lead to the bandgaps.

In this paper, we introduce a dispersion equation for $3 \mathrm{D}$ photonic crystals made of parallel layers of non-overlapping spheres, valid when both wavelength and separation between layers are much larger than the distance between neighbouring spheres. This equation is based on the Korringa-Kohn-Rostoker wave calculation method and can be used to predict the spectral positions of bandgaps in structures made of dispersive spheres. The outline of the paper is as follows. In Section 2, we give details of the approximations involved and the theoretical steps that lead to the aforementioned relation. Then, in Section 3, we apply the simplified equation to investigate structures 
made of dispersive spheres and compare the spectral positions of the bandgaps with those obtained using the MULTEM2 code $[10,11]$, an implementation of the KKR method. It can be used to calculate the transmittance and reflectance of crystal slabs of finite thickness and the band structure of infinite crystals. We also investigate the influence of the constitutive parameters of the spheres and of the embedding medium on the spectral position of the bandgaps. Section 4 concludes the paper.

\section{SIMPLIFIED DISPERSION EQUATION}

A schematic representation of the structure studied and its relevant parameters is shown in Fig. 1. We restrict ourselves to structures made of identical layers of spheres separated by a distance $D=\mathbf{A} \cdot \hat{z}$. Spheres within each layer are located on the sites of a two dimensional lattice. The characteristic distance between the centers of two adjacent spheres is denoted by $d$. Their permittivity and permeability are $\epsilon_{s}$ and $\mu_{s}$, respectively. They are immersed in a homogeneous medium with constitutive parameters $\epsilon$ and $\mu$. A plane wave with wavelength $\lambda=2 \pi / k$ impinges upon the structure.

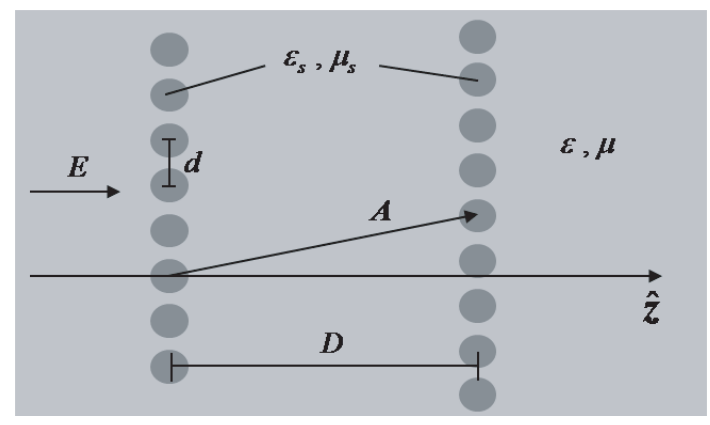

Figure 1. Photonic crystal composed of identical layers of spheres. The incident wavevector is parallel to $\hat{z}$.

The KKR method allows us to obtain the band structure of an infinite photonic crystal made of spheres for any value of the ratios $D / d$ and $\lambda / d$. However, if $D / d \gg 1$ and $\lambda / d \gg 1$, some assumptions can be made and the resulting dispersion equation is much simpler. In what follows we show a brief description of the theoretical steps that lead to a simplified dispersion equation valid in the low frequency limit for structures with $D / d \gg 1$. 
The electromagnetic field at any point of the host medium can be written as a sum of plane waves with wavevectors given by [10]:

$$
\mathbf{k}_{g}^{ \pm}=\left(\mathbf{k}_{\|} ; \pm \sqrt{k^{2}-\left|\mathbf{k}_{\|}\right|^{2}}\right)=\left(\mathbf{q}_{\|}+\mathbf{g} ; \pm \sqrt{k^{2}-\left|\mathbf{q}_{\|}+\mathbf{g}\right|^{2}}\right),
$$

where $\mathbf{k}_{\|}$are the components of $\mathbf{k}_{g}$ parallel to the crystal layers, $\mathbf{g}$ is a reciprocal vector of the 2 D lattice and $\mathbf{q}_{\|}$is a vector in the first Brillouin zone which depends on the direction of incidence. The \pm symbol denotes the sign of the $z$ component of $\mathbf{k}_{g}^{ \pm}$. The amplitudes of the plane waves are obtained using the following eigenvalue equation (similar to Equation (65) of [10]):

$$
\mathbf{U}\left[\begin{array}{l}
{[\mathbf{E}]^{+}} \\
{[\mathbf{E}]^{-}}
\end{array}\right]=\exp \left(\mathbf{i} \mathbf{k}_{\mathbf{b}} \cdot \mathbf{A}\right)\left[\begin{array}{l}
{[\mathbf{E}]^{+}} \\
{[\mathbf{E}]^{-}}
\end{array}\right],
$$

where $\mathbf{k}_{b}$ is the Bloch wavevector and $[\mathbf{E}]^{+}$and $[\mathbf{E}]^{-}$are vectors formed by the amplitudes, for polarizations $S$ (E parallel to the layer) and $P$ (H parallel to the layer), of each plane wave traveling in the $z>0$ and $z<0$ directions, respectively. The matrix $\mathbf{U}$ is the transfer matrix of the crystal. It in turn is the product of the transfer matrix of the crystal layer $(\mathbf{M})$ and the field propagator $(\mathbf{P})$, which accounts for the change of phase of each plane wave as it travels between layers [10]. The sizes of $[\mathbf{E}]^{+},[\mathbf{E}]^{-}$and the transfer matrix $\mathbf{U}$ are determined by the number of plane waves retained in the series expansion of the scattered field.

If the geometrical parameters are such that $D / d \gg 1$, the amplitudes of the evanescent waves decay abruptly from one layer to the other. On the other hand, in the low frequency limit $(\lambda / d \gg 1)$, the only propagating plane waves are those with $\mathbf{k}_{\mathbf{g}=(\mathbf{0}, \mathbf{0})}^{ \pm}$. Therefore, when both conditions hold $(D / d$ and $\lambda / d \gg 1)$ we can keep only the $\mathbf{k}_{\mathbf{g}=(\mathbf{0}, \mathbf{0})}^{ \pm}$wavevectors in Equation (2). Consequently, the matrices $\mathbf{U}$, $\mathbf{M}$ and $\mathbf{P}$ are of dimension $4 \times 4$ and the vectors $[\mathbf{E}]^{+}$and $[\mathbf{E}]^{-}$have only two non-zero components, one for each polarization mode $(S$ or $P)$. Moreover, the non-diagonal terms of matrix $\mathbf{M}$ are zero since no polarization conversion occurs and the problem is separated into two independent polarization modes - one for $S$ and the other for $P$ polarization. Then, the problem reduces to finding the solution of two independent $2 \times 2$ eigenvalue equations, one for each polarization state. Equation (2) can be rewritten as:

$$
\begin{aligned}
& {\left[\begin{array}{cc}
a-b^{2} / a & b / a \\
-b / a & 1 / a
\end{array}\right]\left[\begin{array}{cc}
\exp \left(i \mathbf{k}_{(0,0)}^{+} \cdot \mathbf{A}\right) & 0 \\
0 & \exp \left(i \mathbf{k}_{(0,0)}^{-} \cdot \mathbf{A}\right)
\end{array}\right]\left[\begin{array}{c}
E^{+} \\
E^{-}
\end{array}\right] } \\
= & \exp \left(i \mathbf{k}_{b} \cdot \mathbf{A}\right)\left[\begin{array}{c}
E^{+} \\
E^{-}
\end{array}\right]
\end{aligned}
$$


In this equation, $[E]^{+}$and $[E]^{-}$are unknown complex amplitudes and $a$ and $b$ denote the transmission and reflection coefficients of the monolayer, respectively. They depend on the incident field, the geometry of the 3D lattice and the constitutive parameters of the spheres and of the host medium. From Equation (3), we obtain the following dispersion relation

$$
\begin{aligned}
\cos \left(\mathbf{k}_{b} \cdot \mathbf{A}\right)= & \frac{1}{2 a \exp \left(i \mathbf{k}_{(0,0)}^{+} \cdot \mathbf{A}\right)}\left\{\left[a \exp \left(i \mathbf{k}_{(0,0)}^{+} \cdot \mathbf{A}\right)\right]^{2}\right. \\
& \left.-\left[b \exp \left(i \mathbf{k}_{(0,0)}^{+} \cdot \mathbf{A}\right)\right]^{2}+1\right\} .
\end{aligned}
$$

The above equation can be simplified even further by expressing the real and imaginary parts of the right hand side of (4) in terms of the modules and phases of $a$ and $b$. In the low frequency limit under consideration the absorbance of the spheres is negligible and energy conservation requires that: $1=|a|^{2}+|b|^{2}$. Using this equation and some trigonometric identities we get:

$$
\begin{aligned}
\Re\left[\cos \left(\mathbf{k}_{b} \cdot \mathbf{A}\right)\right]= & \frac{1}{|a|}\left[\cos \left(\delta+\mathbf{k}_{(0,0)}^{+} \cdot \mathbf{A}\right)\right. \\
& \left.-|b|^{2} \cos \left(\phi+\mathbf{k}_{(0,0)}^{+} \cdot \mathbf{A}\right) \cos (\phi-\delta)\right], \\
\Im\left[\cos \left(\mathbf{k}_{b} \cdot \mathbf{A}\right)\right]= & \frac{-|b|^{2} \sin \left(\phi+\mathbf{k}_{(0,0)}^{+} \cdot \mathbf{A}\right) \cos (\phi-\delta)}{|a|},
\end{aligned}
$$

where $a=|a| \exp i \delta$ and $b=|b| \exp i \phi$. A photonic band gap occurs when the right hand side of Equation (6) is non zero, or when the modulus of the right hand side of Equation (5) is greater than unity. For simplicity, in what follows we restrict ourselves to the case of normal incidence. In this case, numerical calculations of $\phi$ and $\delta$ show that the value of $\phi$ is always $\pm \pi / 2+\delta$, the sign \pm depending on the frequency. Deviations from this identity occur in the tenth decimal digit and are negligible. Therefore, we obtain:

$$
\begin{aligned}
& \Im\left[\cos \left(\mathbf{k}_{b} \cdot \mathbf{A}\right)\right]=0, \\
& \Re\left[\cos \left(\mathbf{k}_{b} \cdot \mathbf{A}\right)\right]=\cos \left(\mathbf{k}_{b} \cdot \mathbf{A}\right)=\frac{\cos (\delta+k D)}{|a|} .
\end{aligned}
$$

From Equation (7) we deduce that $\cos \left(\mathbf{k}_{b} \cdot \mathbf{A}\right)$ is always a real number. Equation (8) provides the dispersion relation for the photonic crystal. There are two terms in the argument of the cosine function: the $k D$ term accounts for the change of phase of the plane wave as it travels from one layer to the next and the $\delta$ term is the phase of the 
transmission coefficient of a monolayer and does not depend on the distance between layers. A photonic bandgap occurs when:

$$
\frac{|\cos (\delta+k D)|}{|a|}>1 \text {. }
$$

\section{NUMERICAL RESULTS}

The structure we use for our analysis is a stack of two dimensional square lattices of dispersive spheres embedded in air. The lattice constant of each layer is $d=4 \mathrm{~mm}$, the radius of the spheres is $r=0.3 d$ and the layers are separated a distance $10 d$. We consider two different cases, A and B. Case A corresponds to spheres with non-dispersive permeability $\left(\mu_{s}=1\right)$ and permittivity given by

$$
\epsilon_{s}(\nu)=1+\frac{5^{2}}{0.9^{2}-\nu^{2}}+\frac{10^{2}}{11.5^{2}-\nu^{2}},
$$

where $\nu$ is the frequency measured in $\mathrm{GHz}$. Conversely, case B corresponds to spheres with non-dispersive permittivity $\epsilon_{s}=1$ and permeability

$$
\mu_{s}(\nu)=1+\frac{3^{2}}{0.902^{2}-\nu^{2}} .
$$

The permittivity and permeability functions (10) and (11) have been used in the study of $1 \mathrm{D}$ photonic crystals $[12,13]$. In the following simulations, we will focus on the range of frequencies between $1 \mathrm{GHz}$ and $10 \mathrm{GHz}$.

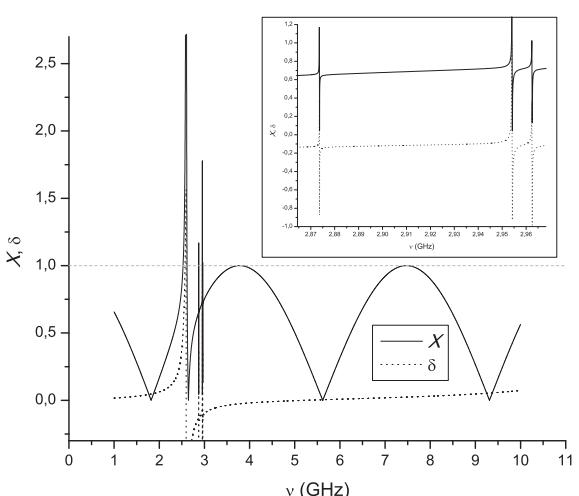

(a)

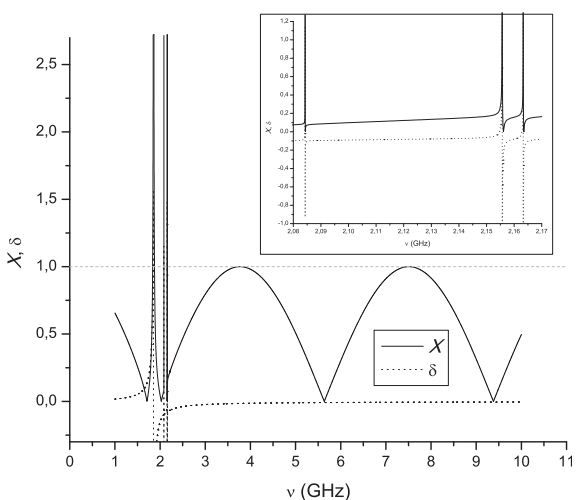

(b)

Figure 2. $X$ vs. $\nu$ (solid line) and $\delta$ vs. $\nu$ (dashed line) for (a) Case A and (b) Case B. 
In Fig. 2, we plot the quantities $X=|\cos (\delta+k D) / a|$ vs. $\nu$ and $\delta$ vs. $\nu$ for case A (Fig. 2(a)) and B (Fig. 2(b)). As we mentioned before, bandgaps are expected at those values of frequencies for which $X$ is greater than 1. A detailed observation of Fig. 2 reveals that there are several values of $\nu$ for which this condition is satisfied. In Fig. 2(a), $X$ exhibits sharp and narrow peaks at $\nu=2.56,2.87,2.95$ and $2.96 \mathrm{GHz}$, approximately (see inset plots). At these frequencies, the phase $\delta$ also has peaks, whereas it is approximately constant in the rest of the spectrum. Therefore, the spectral positions of the peaks in $X$ are mainly associated to the behaviour of $\delta$ at those frequencies. $\delta$ depends on the geometric parameters of the monolayer and the constitutive parameters of the spheres. To investigate this dependence, structures made of layers with other geometries were also studied (not shown). We found that, in the low frequency limit under consideration, the layer geometry does not have a significant influence on the value of $\delta$. This fact suggest that the spectral positions of these bandgaps are intimately connected with the refractive index of the spheres. In Fig. 2(b) a similar behaviour is observed; in this case, the peaks appear at $\nu=1.84,2.08,2.15$ and $2.16 \mathrm{GHz}$, approximately. The condition $X>1$ is also achieved at $\nu=3.78$ and $7.47 \mathrm{GHz}$ in both cases (A and B). However, the behaviour of $X$ at these frequencies is significantly different from the one recently described. In this case, $X$ is barely above the value 1 and is affected mainly by the value of the product $k D$. It can be easily shown that these bandgaps occur at frequencies for which the condition of maximum destructive interference between two successive reflections on the crystal layers is satisfied. Therefore, as expected, these bandgaps appear at the same frequencies for both cases A and B since they do not depend on the constitutive parameters of the spheres. These bandgaps have been extensively investigated in the field of solid state physics using Bragg's law [14-16].

Figure 3 shows the band diagram obtained using the code MULTEM2 for cases A and B. As can be observed, the spectral positions of the bandgaps predicted by expression (9) and those given by MULTEM2 code are in very good agreement. Even though the separation between layers in these examples is $10 d$, good agreement was also found in other cases with separation as low as $5 d$ (not shown here).

To gain further insight into the physical mechanisms that give rise to the various types of bandgaps, we next analyze the dependence of $X$ on the frequency and on the permittivity of the spheres $\left(\epsilon_{s}\right)$ for the parameters of case A. To do so, we calculate $X$ as a function of $\nu$ and $\epsilon_{s}$, considering both quantities as independent variables. In Fig. 4 we present a map showing the pairs $\left(\nu, \epsilon_{s}\right)$ for which $X>1$, 


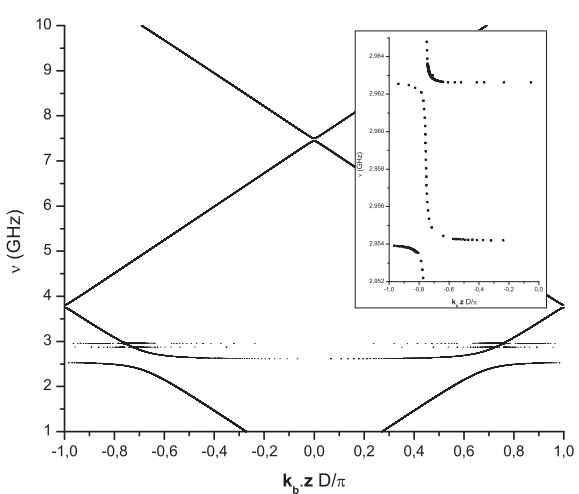

(a)

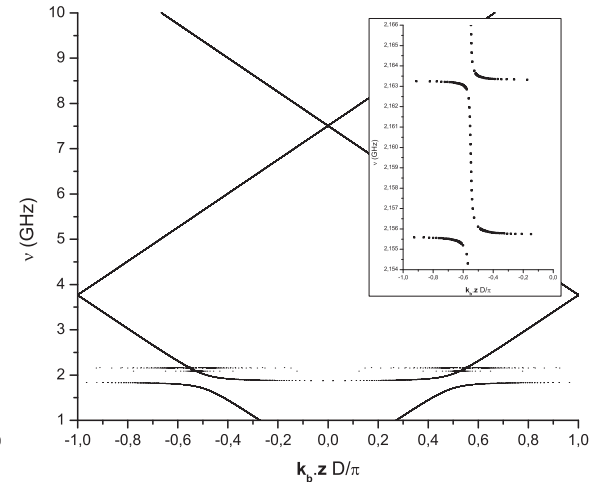

(b)

Figure 3. Band structures obtained with MULTEM2 code for (a) Case A and (b) Case B.

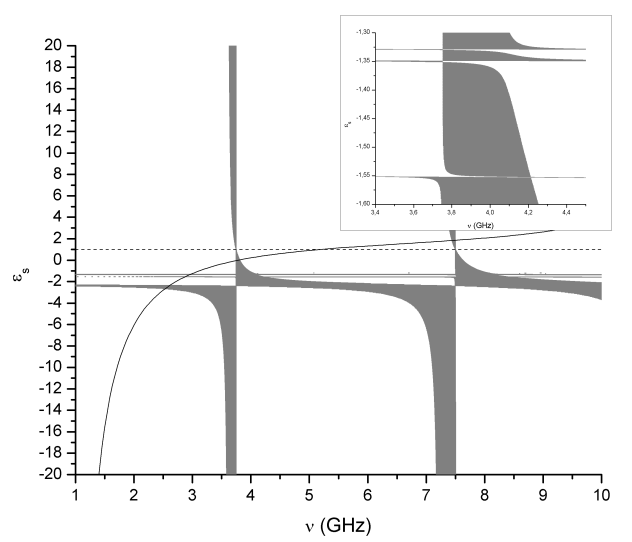

Figure 4. Map showing the pairs $\left(\nu, \epsilon_{s}\right)$ for which $X>1$ for case A. The solid line shows the function $\epsilon_{s}(\nu)$ given by Equation (10).

which are indicated as gray dots. The map makes it possible to easily determine the existence and characteristics of band gaps in crystals with arbitrary $\epsilon_{s}(\nu)$. The black solid curve represents the function $\epsilon_{s}(\nu)$ given by Equation (10).

As can be observed in this figure, there are ranges of frequencies for which bandgaps always exist, regardless of the value of $\epsilon_{s}$. These frequencies are $3.5<\nu<4 \mathrm{GHz}$ and $7<\nu<7.5 \mathrm{GHz}$, approximately. At these frequencies the map exhibits grey vertical stripes that extend over the full range of $\epsilon_{s}$ covered in the example. Comparison with Fig. 2(a) reveal that, as expected, these bandgaps are the ones associated to the interference between layers. Thus, their positions 
depend mainly on the value $k D$ and are almost independent of $\epsilon_{s}$. On the other hand, for $\epsilon_{s} \approx-1.33,-1.35,-1.55$ and -2.5 , approximately, the photonic crystal exhibits bandgaps in the full range of frequencies investigated. Taking into account the dependence of the permittivity with the frequency given by Equation (10), these values of permittivity are achieved when $\nu=2.96,2.95,2.87$ or $2.56 \mathrm{GHz}$, respectively, values which are in perfect agreement with the spectral positions of the bandgaps shown in Fig. 2(a). This is a very interesting result because the bandgaps appear at those frequencies for which the value of $\epsilon_{s}$ coincides with one of the values listed above, regardless of the functional relationship $\epsilon_{s}(\nu)$. For $\epsilon_{s}=1$ the system is essentially an infinite homogeneous medium. All the energy is transmitted through the monolayer. It can be seen in the figure that, as expected, there is no band gap at any frequency for this value of $\epsilon_{s}$. A similar analysis can be done for the structure of case B (not presented here).

To investigate the influence of the host medium on the band structure, we will study two new cases, $\mathrm{C}$ and $\mathrm{D}$. In case $\mathrm{C}$, the spheres are immersed in a medium with $\epsilon=2.5$ and $\mu=1$, whereas in case $\mathrm{D}$, the constitutive parameters of the host medium are $\epsilon=1$ and $\mu=1.4$. For both situations, the permittivity and permeability of the spheres, as well as the other parameters of the structure, are the same as in case A.

In Fig. 5, we show plots of $X$ vs. $\nu$ and $\delta$ vs. $\nu$ for cases C (Fig. 5(a)) and D (Fig. 5(b)). From Fig. 5(a), we deduce that the bandgaps at the lower frequencies appear at $\nu=1.94,2.28,2.37$ and $2.38 \mathrm{GHz}$, approximately. These bandgaps are extremely narrow

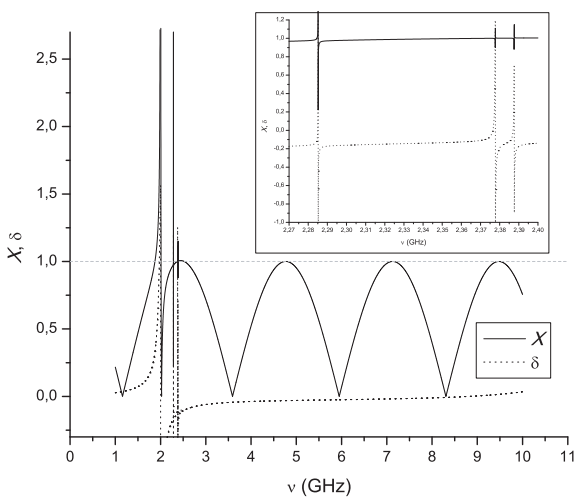

(a)

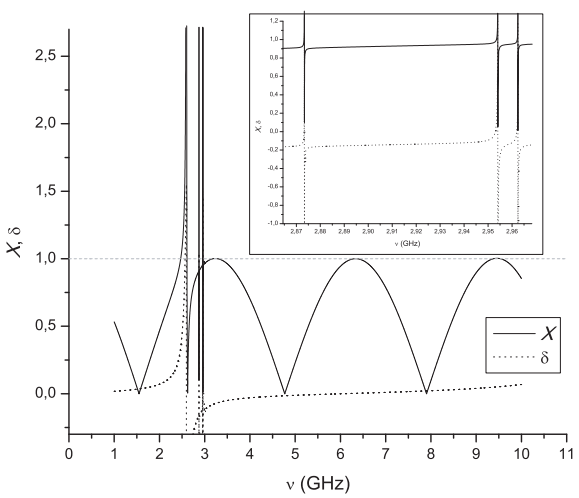

(b)

Figure 5. $X$ vs. $\nu$ (solid line) and $\delta$ vs. $\nu$ (dashed line) for (a) Case $\mathrm{C}$ and (b) Case D. 
and can be better appreciated in the inset plot. At these spectral positions, both $X$ and $\delta$ increase abruptly and exhibit sharp peaks. In addition, there are other bandgaps centered at $\nu=2.45,4.77,7.13$ and $9.47 \mathrm{GHz}$. Comparisons with previous figures show that we can also distinguish two types of bandgaps in these cases. However, the number and positions of the bandgaps are significantly affected by the permittivity of the embedding medium.

On the other hand, if the host medium is magnetic but its permittivity equals 1 (case D), the lowest four gaps occur at, roughly, the same frequencies as in case A. This feature suggests that for the parameters chosen in these examples, the permeability of the host medium does not introduce significant modifications in the spectral positions of the bandgaps. In Fig. 6 we present the band structure obtained using MULTEM2 for cases C (Fig. 6(a)) and D (Fig. 6(b)). As in the previous examples, we also observe in these cases a very good agreement between the position of the bandgaps observed in Fig. 6 and the frequencies for which $X>1$ in Fig. 5 .

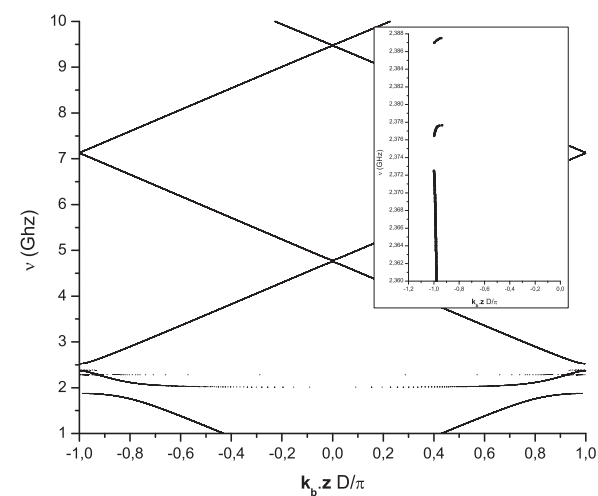

(a)

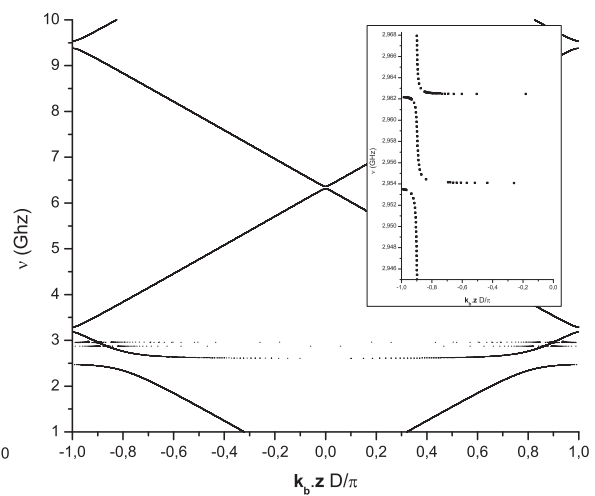

(b)

Figure 6. Band structures obtained with MULTEM2 code for (a) Case C and (b) Case D.

If the spheres are characterized by a dispersive permeability and a fixed value of the permittivity, the results are analogous: the permeability of the host medium (but not its permittivity) affects the number and the positions of the bandgaps.

We conclude that in these structures there are two types of bandgaps: those related to the interference of the waves dispersed by different layers of spheres, and those related to the interference of waves dispersed by the spheres of the same layer. The first type depends mainly on the distance between layers; the second one is 
essentially a single-layer phenomenon, which in the low frequency limit, is primarily dependent on the permittivity and permeability of the spheres. The constitutive parameters of the host medium affect both types of bandgaps.

\section{CONCLUSIONS}

In this paper we have introduced a dispersion equation for $3 \mathrm{D}$ photonic crystals made of parallel layers of non-overlapping spheres, valid when both wavelength and separation between layers are much larger than the distance between neighbouring spheres. This equation can be used to predict the spectral positions of bandgaps in structures made of dispersive spheres. We find that this simplified relation allows us to identify two types of bandgaps: those caused by the interaction of the field with the spheres within a monolayer and those due to the destructive interference between the waves reflected on the crystal layers. The constitutive parameters of the embedding medium affect both types of bandgaps. Perfect agreement between the spectral positions of bandgaps predicted by our simplified equation and those obtained with MULTEM2 code was observed. We have shown examples to illustrate the influence of the constitutive parameters of the spheres on the spectral positions of bandgaps, for spheres immersed in dielectric and magnetic homogeneous media. The $\epsilon_{s}-\nu$ maps allow us to easily determine the existence and characteristics of bandgaps in crystals with arbitrary dielectric function.

\section{ACKNOWLEDGMENT}

F. G. acknowledges financial support from CONICET, UBA and ANPCyT (PRH74). M. I. acknowledges financial support from CONICET (grant PIP 112-200801-01880), ANPCyT (ANPCYT-BID grant No. 1728/OC-AR06-01785), and UBA (grant X208 and X848).

\section{REFERENCES}

1. Yablonovitch, E., "Inhibited spontaneous emission in solid-state physics and electronics," Phys. Rev. Lett., Vol. 58, No. 20, 20592062, 1987.

2. Sakoda, K., Optical Properties of Photonic Crystals, SpringerVerlag, Berlin, 2001.

3. Joannopoulos, J. R., R. D. Meade, and J. N. Winn, Photonic Crystals, Princeton University Press, Princeton, 1995. 
4. Ho, K. M., C. T. Chan, and C. M. Soukoulis, "Existence of a photonic gap in periodic dielectric structures," Phys. Rev. Lett., Vol. 65, No. 25, 3152-3155, 1990.

5. Ohtaka, K., "Scattering theory of low-energy photon diffraction," J. Phys. C, Vol. 13, No. 4, 667, 1980.

6. Modinos, A., "Scattering of electromagnetic waves by a plane of spheres-formalism," Physica A, Vol. 141, No. 2, 575-588, 1987.

7. Stefanou, N., V. Karathanos, and A. Modinos, "Scattering of electromagnetic waves by periodic structures," J. Phys.: Condens. Matter, Vol. 4, No. 36, 7389, 1992.

8. Dorado, L. A., R. A. Depine, and H. Miguez, "Effect of extinction on the high-energy optical response of photonic crystals," Phys. Rev. B, Vol. 75, No. 24, 241101, 2007.

9. Dorado, L. A., R. A. Depine, G. Lozano, and H. Miguez, "Interplay between crystal-size and disorder effects in the highenergy optical response of photonic crystal slabs," Phys. Rev. B, Vol. 76, No. 24, 245103, 2007.

10. Stefanou, N., V. Yannopapas, and A. Modinos, "Heterostructures of photonic crystals: Frequency bands and transmission coefficients," Comput. Phys. Commun., Vol. 113, No. 1, 49-77, 1998.

11. Stefanou, N., V. Yannopapas, and A. Modinos, "MULTEM 2: A new version of the program for transmission and band-structure calculations of photonic crystals," Comput. Phys. Commun., Vol. 132, No. 1, 189-196, 2000.

12. Monsoriu, J., R. A. Depine, M. L. Martinez Ricci, and E. Silvestre, "Interaction between non-Bragg band gaps in 1D metamaterial photonic crystals," Opt. Express, Vol. 14, No. 26, 12958, 2006.

13. Li, J., L. Zhou, C. T. Chan, and P. Sheng, "Photonic band gap from a stack of positive and negative index materials," Phys. Rev. Lett., Vol. 90, No. 8, 083901, 2003.

14. Ashcroft, N. W. and N. D. Mermin, Solid State Physics, Saunders College Publishing, Philadelphia, 1976.

15. Kapitza, P. L. and A. M. Dirac, "The reflection of electrons from standing light waves," Mathematical Proceedings of the Cambridge Philosophical Society, Vol. 29, No. 2, 297-300, 1933.

16. Zachariasen, W. H., Theory of X-ray Diffraction in Crystals, Courier Dover Publications, New York, 2004. 